\title{
THE ZYGMUND CONDITION FOR BLOCH FUNCTIONS IN THE BALL IN $C^{n}$
}

\author{
JOSEPH. A. CIMA AND BARNET M. WEINSTOCK ${ }^{1}$
}

\begin{abstract}
ABstruct. In this paper we prove the equivalence of the Bloch condition for a holomorphic function $f$ on the ball $B_{n}$ with the Zygmund second difference condition for a suitable primitive $F$ of $f$.
\end{abstract}

Introduction. If $B_{1}$ is the open unit disc in the complex plane and $f$ is holomorphic on $B_{1}$, we say that $f$ is a Bloch function if there exists a positive number $M$ such that

$$
\left|f^{\prime}(z)\right|\left(1-|z|^{2}\right)<M
$$

for all $z \in B_{1}$. When equipped with an appropriate norm the linear space of Bloch functions becomes a nonseparable Banach space. There are many equivalent conditions that a function can satisfy to be a Bloch function (see Pommerenke [2] or Cima [1]). In a recent thesis, Richard Timoney [4] has done an exhaustive study of properties of Bloch functions on domains in $C^{n}$. In particular his work includes the theory of Bloch functions on the ball

$$
B_{n}=\left\{z \in C^{n}:\|z\|=\sqrt{\left|z_{j}\right|^{2}}<1\right\} \text {. }
$$

He has shown that all the known characterizations, save two, that are equivalent for the case of $n=1$ are valid for $n>1$. One of these two characterizations is the second difference condition of Zygmund [5]. We will establish the equivalence of this condition for the $B_{n}$ case.

1. Preliminaries. Assume $f$ is a holomorphic function of $B_{n} \rightarrow C$. For $u$ and $v$ vectors in $C^{n}, z \in B_{n}$ and $\langle u, v\rangle=\sum_{j=1}^{n} u_{j} \bar{v}_{j}$ the Bergman metric is given by

$$
H_{z}(u, \bar{v})=\left(\frac{n+1}{2}\right)\left[\frac{\left(1-\|z\|^{2}\right)\langle u, \bar{v}\rangle+\langle u, \bar{z}\rangle\langle z, \bar{v}\rangle}{\left(1-\|z\|^{2}\right)^{2}}\right] .
$$

For each $z \in B_{n}$ define

$$
Q_{f}(z) \equiv \sup \left\{\left|\left(\nabla_{z} f\right)(x)\right| / H_{z}(x, \bar{x})^{1 / 2} ; x \in C^{n}, x \neq 0\right\}
$$

where $\left(\nabla_{z} f\right)(x)=\sum_{j=1}^{n}\left(\partial f / \partial z_{j}\right)(z) x_{j}$.

Received by the editors April 27, 1979 and, in revised form, June 4, 1979; presented to the Society, August 25, 1979.

AMS (MOS) subject classifications (1970). Primary 32A30, 46E15.

$K e y$ words and phrases. Bloch function, several complex variables, Zygmund condition.

${ }^{1}$ Partially supported by National Science Foundation Grant MCS78-02912. 
Definition 1. A holomorphic function $f: B_{n} \rightarrow C$ is called $a$ Bloch function if $\sup \left\{Q_{f}(z): z \in B_{n}\right\}<\infty$.

In considering this definition, a certain amount of pathology immediately enters. In fact one observes by studying the metric that if $f$ is a Bloch function, then the growth of its directional derivative in the radial direction is $O\left(\left(1-\|z\|^{2}\right)^{-1}\right)$, whereas the growth in directions orthogonal to the radial direction is only $O\left(\left(1-\|z\|^{2}\right)^{-1 / 2}\right)$. Timoney [4] shows that the Bloch condition is equivalent to the following condition:

$$
\sup \left\{\left\|\nabla_{z} f\right\|\left(1-\|z\|^{2}\right) ; z \in B_{n}\right\}<\infty .
$$

Assume $F$ is in the ball algebra of $B_{1}$, i.e., $F$ is continuous on $\bar{B}_{1}$ and holomorphic in $B_{1}$. Further assume that the boundary values of $F$ satisfy

$$
\left|F\left(e^{i(\theta+h)}\right)+F\left(e^{i(\theta-h)}\right)-2 F\left(e^{i \theta}\right)\right|<A|h|
$$

for all real numbers $h$ and some positive number $A$, independent of $\theta$. In [5] it was shown that a function $f$, holomorphic on $B_{1}$, satisfies the Bloch condition if and only if its primitive $F(z) \equiv \int_{0}^{z} f(\zeta) d \zeta$ is in the ball algebra of $B_{1}$ and satisfies condition (1.2).

We consider in this note $C^{1}$ curves $\gamma$ mapping $\mathbf{R} \rightarrow \partial B_{n}$ such that $\left|\gamma^{\prime}(t)\right|=1$ for all $t$. We refer to these as normalized $C^{1}$ curves. With this class of curves in mind we make the following definition.

DEFINITION 1.2. Let $F$ be in the ball algebra of $B_{n}$. We say that $F$ satisfies condition $\Lambda_{*}\left(\partial B_{n}\right)$ if there exists a positive number $A$ such that for all normalized $C^{1}$ curves $\gamma$ in $B_{n}$,

$$
|F(\gamma(t+h))+F(\gamma(t-h))-2 F(\gamma(t))|<A|h|
$$

for all $t$ and $h$ in $\mathbf{R}$.

Finally, if $f$ is holomorphic on $B_{n}$ with expansion in terms of homogeneous polynomials given by $f(z)=\sum_{k=0}^{\infty} F_{k}(z)$ define the radial derivative $R f$ of $f$ by the formula

$$
\Re f(z) \equiv \sum_{k=1}^{\infty} k F_{k}(z) .
$$

2. The principal result. If we are given a function $f$ holomorphic on $B_{n}$, define a function

$$
F(z)=(\mathscr{P} f)(z) \equiv \int_{0}^{1} f(t z) d t
$$

One checks that $\mathscr{R}(\mathscr{P} f)(z)=\Re F(z)=f(z)-F(z)$.

THEOREM 1. A function $f$ holomorphic on $B_{n}$ is in the Bloch space of $B_{n}$ if and only if $\mathscr{P} f$ satisfies the $\Lambda_{*}\left(\partial B_{n}\right)$ condition.

Proof. Assume first that $f$ is a Bloch function. For $a \in \partial B_{n}$ the slice functions are defined by $f_{a}(\lambda)=f(\lambda a), \lambda \in B_{1}$. Since

$$
(\mathscr{P} f)_{a}(\lambda) \equiv F_{a}(\lambda)=\frac{1}{\lambda} \int_{0}^{\lambda} f_{a}(\zeta) d \zeta
$$


we see that each $F_{a}$ is in $\Lambda_{*}\left(\partial B_{1}\right)$. Also each member of the family $\left\{F_{a} ; a \in \partial B_{n}\right\}$ has its oscillation $\omega\left(F_{a}, \delta\right)=O(\delta \log \delta)$, uniformly in $a . F$ can be extended to a function on $\bar{B}_{n}$ by using the values on the slices. Now with $b \in \partial B_{n}, 0<r<1$,

$$
|F(a)-F(b)|<M(1-r) \log (1-r)+\left|F_{a}(r)-F_{b}(r)\right| .
$$

This shows that $F$ is continuous at $a$ and hence is in the ball algebra.

Now fix a normalized $C^{1}$ curve $\gamma$ with range in $\partial B_{n}$ and let $h>0$ be given. If $g(t)$ is any function defined on R set $A g(t)=g(t+h)-g(t)$. With $r=1-h$ we write

$$
F(\gamma(t))=(F(\gamma(t))-F(r \gamma(t)))+(F(r \gamma(t)))
$$

and show that $A^{2}$ of each expression in parentheses is $O(h)$, independent of $t$. Since $|f(z)|=O(\log (1-|z|))$ one easily verifies that

$$
F(\gamma(t))-F(r \gamma(t))=(1-r) f(r \gamma(t))+\int_{r}^{1}(1-s) \nabla f(s \gamma(\theta)) \circ \gamma(\theta) d s .
$$

The integral in this equality is $O(h)$. Also

$$
A f(r \gamma(t))=\int_{0}^{h} \nabla f(\zeta) \circ \zeta^{\prime}=O(1)
$$

where $\zeta(p)=r \gamma(t+p)$. Hence

$$
A^{2}[F(\gamma(t))-F(r \gamma(t))]=O(h)
$$

uniformly in $t$. The expression $A^{2} F(r \gamma(t))$ involves three terms:

$$
\begin{gathered}
r \int_{0}^{h}\left(\gamma^{\prime}(t+p)-\gamma^{\prime}(t)\right) \circ \nabla F(r \gamma(t+p)) d p \\
r \int_{0}^{h} \gamma^{\prime}(t) \circ(\nabla F(r \gamma(t+p))-\nabla F(r \gamma(t-p))) d p \\
r \int_{0}^{h}\left(\gamma^{\prime}(t)-\gamma^{\prime}(t-p)\right) \circ \nabla F(r(\gamma(t-p))) d p
\end{gathered}
$$

By the definition

$$
\left|\frac{\partial F}{\partial z_{j}}(r \gamma(t))\right|=\left|r \int_{0}^{1} \nabla f(r u \gamma(t)) \circ \gamma^{\prime}(t) u d u\right|<M \cdot|\log h| .
$$

Thus, expressions (2.2) and (2.4) are $O(1)$ uniformly in $t$. Similarly

$$
\|\nabla F(r \gamma(t))-\nabla F(r \gamma(s))\|=O(\log (1-r))
$$

and hence (2.3) is $O(1)$.

For the converse we observe that for each $0<\alpha<1$, the space $\Lambda_{\alpha}\left(B_{1}\right)$ of functions in the ball algebra with boundary values in the Lip $\alpha$ space contains $\Lambda_{*}\left(B_{1}\right)$. Further, bounded subsets of $\Lambda_{*}$ are bounded in $\Lambda_{\alpha}$. Fix $z=r e_{1}$ in $B_{n}$ with $\left\|e_{1}\right\|=1,0<r<1$, and let $\left\{e_{j}\right\}_{j=1}^{n}$ be an orthonormal basis for $C^{n}$. Let $D_{j}$ be the derivative in the $e_{j}$ direction. We can apply a result of Rudin [3] to draw the following conclusions. Since $\left\{F_{w}\right\}$ is a norm-bounded subset of $\Lambda_{1 / 2}\left(B_{1}\right)$,

$$
(\Re F)(z)=O\left((1-\|z\|)^{-1 / 2}\right)
$$

and

$$
D_{j} f(z)=O\left((1-\|z\|)^{-1}\right), \quad 2<j<n .
$$


Since $F_{e_{1}}(r)+(\mathscr{R} F)_{e_{1}}(r)=f_{e_{1}}(r)$ we apply the one variable result to conclude

$$
D_{1} f(z)=D f_{e_{1}}(r)=O\left((1-\|z\|)^{-1}\right) \text {. }
$$

The estimates are uniform.

The referee has pointed out that our proof yields the following equivalences.

Proposimon 1. A homolomorphic function $f: B_{n} \rightarrow C$ is a Bloch function if and only if the slice functions $F_{a}=(\mathscr{P} f)_{a}, a \in \partial B_{n}$, are uniformly bounded in $\Lambda_{*}\left(\partial B_{1}\right)$.

Proof. From [4] a function $f: B_{n} \rightarrow C$ is a Bloch function if and only if

$$
\operatorname{Sup}_{z \in B_{n}}|(\Re f)(z)|\left(1-\|z\|^{2}\right)<\infty .
$$

The functions $(\mathscr{P} f)_{a}, a \in \partial B_{n}$, are uniformly bounded in $\Lambda_{*}\left(\partial B_{1}\right)$ if and only if

$$
\operatorname{Sup}_{|z|<1, a \in \partial B_{n}}\left|\left[(\mathscr{P} f)_{a}\right]^{\prime \prime}(z)\right|\left(1-|z|^{2}\right)<\infty .
$$

A computation with (2.1) shows that

$$
\begin{aligned}
{\left[(\mathscr{P} f)_{a}\right]^{\prime \prime}(z) } & =\frac{1}{z^{2}}(\Re f)_{a}(z)-\frac{2}{z^{2}}\left[f_{a}(z)-(\mathscr{P} f)_{a}(z)\right] \\
& =\frac{1}{z^{2}}(\Re f)_{a}(z)-\frac{2}{z}\left[(\mathscr{P} f)_{a}\right]^{\prime}(z) .
\end{aligned}
$$

It is clear that (2.5) and (2.6) are equivalent.

Proposition 2. A holomorphic function $F: B_{n} \rightarrow C$ is in $\Lambda_{*}\left(\partial B_{n}\right)$ if and only if the slice functions $F_{a}, a \in \partial B_{n}$, are uniformly bounded in $\Lambda_{*}\left(\partial B_{1}\right)$.

PROOF. This follows from Rudin's result [3] and the proof of Theorem 1.

A comment is in order. If one considers the latter half of the proof of Theorem 1, one sees that $\mathscr{P f}=F$ is much more smooth on curves $\gamma(t)$ whose tangents lie in the "complex tangential direction." However, the function $f(z)=\log \left(1-z_{1}^{2}-z_{2}^{2}\right)$ achieves the proper growth estimate on curves $\gamma(t)=e^{i t w}(\|w\|=1)$ whose tangents lie in the real direction.

\section{REFERENCES}

1. J. A. Cima, The basic properties of Bloch functions, Internat. J. Math. and Math. Sci. 2 (1979).

2. Ch. Pommerenke, On Bloch functions, J. London Math. Soc. 2 (1970), 689-695.

3 W. Rudin, Holomorphic Lipschitz functions in balls, Comment. Math. Helv. 53 (1978), 143-147.

4. R. Timoney, Bloch functions in several complex variables, Thesis, University of Illinois, 1978.

5. A. Zygmund, Smooth functions, Duke Math. J. 12 (1945), 47-76.

Department of Mathematics, Universtty of North Caroldna, Chapel Hill, North Carolna 27514.

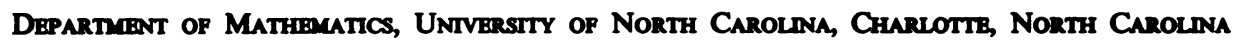
28223 